https://doi.org/10.15407/ujpe65.10.865

O.O. BOLIASOVA*, V.N. KRIVORUCHKO

O.O. Galkin Donetsk Institute for Physics and Engineering, Nat. Acad. of Sci. of Ukraine

(46, Nauky Av., Kyiv 03028, Ukraine; e-mail: *ol.boliasova@gmail.com)

\title{
LONGITUDINAL SPIN DYNAMICS IN THE HEISENBERG ANTIFERROMAGNET: TWO-MAGNON EXCITATIONS
}

\begin{abstract}
Understanding the ultrafast spin dynamics in magnetically ordered materials is important for the comprehenssion of fundamental limits in spin-based magnetic electronics - magnonics. We have studied a microscopic model of magnetization dynamics in a two-sublattice antiferromagnet with the emphasis on longitudinal spin excitations. The diagrammatic technique for spin operators has been used to overcome limitations typical of phenomenological approaches. The graphical representations of spin wave propagators allow us to summing up the infinite series of distinctive diagrams. Its sum is transformed into an analytic expression for the longitudinal spin susceptibility $\chi^{z z}(\mathbf{q}, \omega)$ applicable in all regions of the frequency $\omega$ and wave vector $\mathbf{q}$ spaces beyond the hydrodynamical and critical regimes. It is found that the longitudinal magnetization dynamics consists of two types of excitations, which have different dependences on the temperature and wave vector $\mathbf{q}$. The obtained result could be important for understanding the physics of nonequilibrium magnetic dynamics under the effect of ultrafast laser pulses in antiferromagnetic materials.
\end{abstract}

Keywords: longitudinal magnetization dynamics, antiferromagnet, magnonics.

\section{Introduction}

The concept of spin wave (SW) as a low-energy collective excitation is a reasonable approximation for the description of the transverse spin dynamics at low temperatures and energies. The longitudinal spin excitation demonstrates a more complex behavior. The study of the longitudinal spin dynamics within the framework of the Heisenberg model was the subject of numerous theoretical and experimental works (see, e.g., [1-8] and references therein). According to the pioneering paper Vaks, Larkin, and Pikin (VLP) [9], the longitudinal spin mode arises as a result of the virtual processes of coherent creation and annihilation of SWs (magnons). More recently, the dynamic longitudinal spin susceptibilities of the Heisenberg ferromagnet and ferrimagnet have been studied using the diagrammatic technique for spin operators. For a ferromagnet [2], the uniform longitudinal susceptibility is zero, $\chi^{z z}(0, \omega)=0$, for a nonzero frequency $\omega \neq 0$. This is a consequence of the total spin conservation law. Generally, the longitudinal spin excitations eventuate as the virtual processes of creation

(C) O.O. BOLIASOVA*, V.N. KRIVORUCHKO, 2020

ISSN 2071-0194. Ukr. J. Phys. 2020. Vol. 65, No. 10 and annihilation of transverse SWs at the frequency $\omega(\mathbf{q})=\varepsilon_{\mathbf{q}}-\varepsilon_{\mathbf{q} \pm \mathbf{k}}$. Excitation processes are controlled by the occupation factor, determined through the Bose distribution function which makes the SWs with wave vector $\mathbf{q} \sim 0$ to be dominant. For a twosublattice ferrimagnet [8], the dynamics of longitudinal spin components is due to a few virtual processes: (i) acoustic $\omega_{a}(\mathbf{q})=\varepsilon_{i, \mathbf{q}}-\varepsilon_{i, \mathbf{q} \pm \mathbf{k}}$ and (ii) exchange $\omega_{e x}(\mathbf{q})=\varepsilon_{i, \mathbf{q}}+\varepsilon_{j, \mathbf{q} \pm \mathbf{k}}$ of transverse SWs $(i \neq j, i, j=1,2)$. The existence of the exchange longitudinal mode at such frequencies can result in a new form of excitation behavior in a ferrimagnetic system, which is important for understanding the physics of non-equilibrium magnetic dynamics under the effect of ultrafast laser pulses in multisublattice magnetic materials. The ultrafast spin dynamics for ferrimagnets close to the spin compensation point is discussed in [10].

Recent studies have shown the possibility to control the magnetization dynamics of magnetic materials by optical methods on a femtosecond time scale [11-15]. Those open new opportunities for the practical application of magnetic materials and antiferromagnets (AFMs), in particular. In the case of AFMs, 
we have a few advantages. First of all, the spin resonance of AFMs is in the terahertz range, that gives the possibility to make magnonics devices faster than on the base of ferromagnets $[13,15]$. Moreover, AFMs in memory devices are resistant to perturbations from external magnetic and electric fields $[16,17]$. It is also important that the antiferromagnetic order is present in a lot of dielectrics, metals, and semiconductors. However, despite active experimental and theoretical studies, the longitudinal magnetization dynamics in AFMs is yet not well understandable.

The longitudinal spin dynamics was studied experimentally in AFM $\mathrm{MnF}_{2}$ [18]. Using the polarized neutron scattering, the authors showed that the spectrum of longitudinal excitations consists of two branches. One of them is located below the energy excitation of one magnon and represents the excitation of a magnon and the absorption of another one. The second branch is located above of the onemagnon energy and corresponds to the simultaneous absorption or excitation of two magnons [18]. The Monte Carlo calculation for anisotropic AFMs also shows the presence of two types of the longitudinal propagative excitations, which are entirely multiple SWs in nature. The intensity of two-magnon excitations increases with decreasing the wave vector q; with increasing the temperature, these two peaks broaden [19].

Here, we will study the spin dynamics of the Heisenberg AFM quantum model using the diagrammatic technique for the spin operators. We will discuss the conditions of applicability of the proposed calculation method, its efficiency and accordance to the task at hand, and we will analyze more thoroughly the properties of the systems under consideration. The preliminary results of this study can be found in Ref. [20].

This work is structured as follows. The next section clarifies the model used. The third and fourth ones give more detailed representations of the longitudinal and transverse Green functions (GFs), respectively. They are necessary for calculating the magnetic susceptibility $\chi^{z z}(\mathbf{q}, \omega)$, which determines the longitudinal dynamics of an AFM. The following section summarizes the calculations, and its subsections describe the longitudinal dynamic as two-transversemagnon processes in more details. We end with Discussion. Some mathematical details are presented in Appendices.

\section{The Model}

We consider a simple anisotropic model of a twosublattice AFM. In the absence of any external influences, the Heisenberg Hamiltonian is formed purely by exchange, still anisotropic, interactions:

$H=\sum_{\mathbf{f}, \mathbf{g}}\left[\frac{1}{2} J_{\mathbf{f g}}\left(S_{\mathbf{f}}^{+} S_{g}^{-}+S_{\mathbf{f}}^{-} S_{\mathbf{g}}^{+}\right)+J_{\mathbf{f g}}^{z} S_{\mathbf{f}}^{z} S_{\mathbf{g}}^{z}\right]$.

Here, $\mathbf{S}_{\mathbf{f}}$ and $\mathbf{S}_{\mathbf{g}}$ are the spin operators on the $\mathbf{f}(\mathbf{g})$-th sites of sublattices 1 and 2, respectively. The circular spin operators $S^{ \pm}$are $S^{ \pm}=S^{x} \pm i S^{y}, J_{\mathrm{fg}}$ is the exchange interaction between spin $x-y$ components, $J_{\text {fg }}^{z}$ stands for the exchange integral between the $z$ components of spins. We suggest that $\left|\mathbf{S}_{f}\right|=\left|\mathbf{S}_{g}\right|=S$ and $J_{\mathrm{fg}}^{z}>J_{\mathrm{fg}}>0$, i.e., the sublattices are in the antiparallel orientation along the $z$-axis in equilibrium.

We represent Hamiltonian (1) as a sum of three terms $H=E_{0}+H_{0}+H_{\text {int }}$. The first term is the ground-state energy $E_{0}=-J_{0}^{z}\left\langle S^{z}\right\rangle^{2} N$. Here, $N$ is a number of magnetic primitive cells, $J_{0}^{z}=J_{q=0}^{z}$ is the Fourier transform of the exchange interaction, $H_{0}$ stands for the Hamiltonian of the molecular field of a standard structure: $H_{0}=y \sum_{\mathbf{f}, \mathbf{g}}\left(S_{\mathbf{f}}^{z}-S_{\mathbf{g}}^{z}\right)$, where $y=\left\langle S^{z}\right\rangle J_{0}^{z}$. The interaction Hamiltonian $H_{\text {int }}$ has the form:

$$
\begin{aligned}
& H_{\mathrm{int}}=\sum_{\mathbf{f}, \mathbf{g}}\left[\frac{1}{2} J_{\mathrm{fg}}\left(S_{\mathbf{f}}^{+} S_{g}^{-}+S_{\mathbf{f}}^{-} S_{\mathbf{g}}^{+}\right)+\right. \\
& \left.+J_{\mathrm{fg}}^{z}\left(S_{\mathbf{f}}^{z}-\left\langle S^{z}\right\rangle\right)\left(S_{\mathrm{g}}^{z}+\left\langle S^{z}\right\rangle\right)\right] .
\end{aligned}
$$

In the zero-order approximation of a self-consistent field, we have $\left\langle S_{\mathbf{f}}^{z}\right\rangle^{(0)}=-\left\langle S_{\mathbf{g}}^{z}\right\rangle^{(0)}=\left\langle S^{z}\right\rangle^{(0)}=b\left(\beta y_{0} S\right)$, $b(x)=S B_{S}(x)$ and $B_{S}(x)$ is the Brillouin function, $y_{0}=b J_{0}^{z}$, and $\beta^{-1}=T$ is the temperature.

Within the microscopic (Green function method) approach, the study of system's longitudinal magnetization dynamics is reduced to a calculation of the dynamic susceptibility $\chi^{z z}(\mathbf{q}, \omega)$, being a function of the frequency $\omega$ and the momentum $\mathbf{q}[21,22]$. The calculation of system's longitudinal susceptibility $\chi^{z z}(\mathbf{q}, \omega)$ corresponds to a calculation of the retarded longitudinal spin GF $G_{\text {tot }}^{z z(R)}(\mathbf{q}, \omega)[21,22]$ :

$$
\begin{aligned}
& \chi^{z z}(\mathbf{q}, \omega)=\left.\left\langle\left\langle\widehat{T} M_{\mathrm{tot}}^{z}(t) \mid M_{\mathrm{tot}}^{z}(0)\right\rangle\right\rangle\right|_{\mathbf{q}, \omega}= \\
& =-g^{2} \frac{\mu_{\mathrm{B}}^{2}}{\nu_{0}} G_{\mathrm{tot}}^{z z(R)}(\mathbf{q}, \omega) .
\end{aligned}
$$


Here, $M_{\text {tot }}^{z}$ is the $z$-component of the total magnetization $\mathbf{M}_{\text {tot }}=g \mu_{\mathrm{B}}\left(\mathbf{S}_{f}+\mathbf{S}_{g}\right), g-g$-factor, $\mu_{\mathrm{B}}$ is the Bohr magneton, and $\nu_{o}$ stands for the volume of a primitive magnetic cell. In the ground state of an AFM, the mean value of the magnetic primitive cell magnetization $\mathbf{M}$ is egual to $\left\langle M_{\text {tot }}\right\rangle=0$. The symbol $\langle\langle\ldots\rangle\rangle_{\mathbf{q}, \omega}$ denotes the Fourier transform of the trace of $\rho_{0}(\ldots)$ with $\rho_{0}=\exp \left(-\beta H_{0}\right) / S p\left(\exp \left(-\beta H_{0}\right)\right) ; \widehat{T}$ stands for the time-ordering operator. The theorems are proved that the poles of the retarded GFs correspond to the frequencies of magnetization excitations that are transverse magnetization oscillations of the spins or ordinary SWs and longitudinal spin oscillations. In turn, the retarded GFs of the system can be obtained from the temperature GFs by the analytic continuation from the Matsubara frequencies $i \omega_{n}$ onto the real axis $i \omega_{n} \rightarrow \omega+i \delta,(\delta \rightarrow 0)$ (for more details, see, e.g., books $[21,22])$.

In our case of a two-sublattice system, the total GF $G_{\text {tot }}^{z z(R)}(\mathbf{q}, \omega)$ can be reduced to four sublattice longitudinal GFs $G_{i j}^{z z}\left(\mathbf{q}, i \omega_{n}\right)(\mathrm{i}, \mathrm{j}=1,2)$ as follows:

$G_{\mathrm{tot}}^{z z}\left(\mathbf{q}, i \omega_{n}\right)=\left.\left\langle\left\langle\widehat{T}\left(\delta S_{\mathbf{f}}^{z}-\delta S_{\mathbf{g}}^{z}\right) \mid\left(\delta S_{\mathbf{f}}^{z}-\delta S_{\mathbf{g}}^{z}\right)\right\rangle\right\rangle\right|_{\mathbf{q}, \omega}$,

where $i \omega_{n}=i 2 \pi n T(n=0, \pm 1, \pm 2, \ldots)$ is the Matsubara frequency and $\delta S_{i}^{z}=S_{i}^{z}-\left\langle S_{i}^{z}\right\rangle(i=\mathbf{q}, \mathbf{f})$. Below, we use the notation $q=\left\{\mathbf{q}, i \omega_{n}\right\}$ and rewrite the above expression as

$G_{\mathrm{tot}}^{z z}(q)=\left[G_{11}^{z z}(q)-\left(G_{12}^{z z}(q)+G_{21}^{z z}(q)\right)+G_{22}^{z z}(q)\right]$.

Thus, the calculation of the dynamic susceptibility $\chi^{z z}(\mathbf{q}, \omega)$ reduces to the calculation of the sublattice longitudinal GFs $G_{i j}^{z z}\left(\mathbf{q}, i \omega_{n}\right)$.

\section{Green's Functions of Longitudinal Spin Components}

To calculate the sublattice GFs, we use the Larkin equation [9], see also [21, 22]. The graphical representation of the equations for the longitudinal GFs $G_{11}^{z z}(q)$ and $G_{21}^{z z}(q)$ are shown in Fig. 1. Here, the loops of thick lines represent the irreducible parts $\Sigma_{i j}$, and the wavy line matches the interaction $J_{\mathbf{q}}^{z}$. In terms of the diagrammatic technique, the quantity $\Sigma_{i j}^{z}(q)$ is called the irreducible (by Larkin's method of choosing the irreducible diagrams) parts $[21,22]$. Note that the irreducibility is understood here in the sense that $\Sigma_{i j}^{z}(q)$ is represented by the collection of all diagrams from the series for $G_{i j}^{z z}(q)$ that cannot be cut

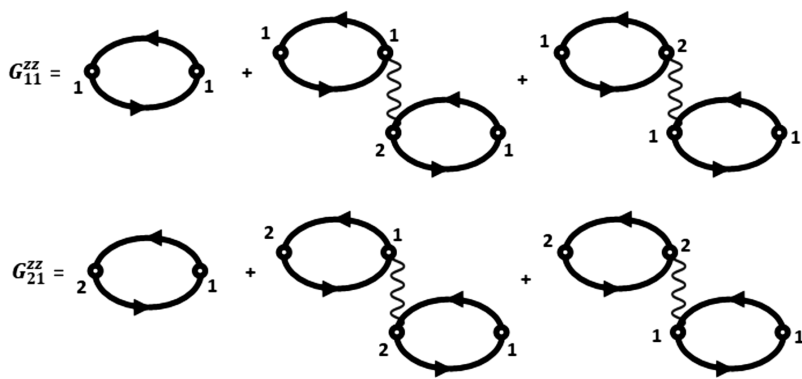

Fig. 1. The graphical presentation of the longitudinal GFs $G_{11}^{z z}$ and $G_{21}^{z z}$

across a line of interaction $J_{\mathbf{q}}$ or $J_{\mathbf{q}}^{z}$. We recall that the graph series for the $G_{11}^{z z}(q)$ and $G_{21}^{z z}(q)$ functions of a two-sublattice AFM can be presented analyticly in the form:

$$
\begin{aligned}
& G_{11}^{z z}(q)= \\
& =\frac{\Sigma_{11}^{z}(q)}{\left[1-J_{\mathbf{q}}^{z} \Sigma_{12}^{z}(q)\right]\left[1-J_{\mathbf{q}}^{z} \Sigma_{21}^{z}(q)\right]-\left(J_{q}^{z}\right)^{2} \Sigma_{11}^{z}(q) \Sigma_{22}^{z}(q)}
\end{aligned}
$$

$G_{21}^{z z}(q)=$

$$
=\frac{\left[1-J_{\mathbf{q}}^{z} \Sigma_{12}^{z}(q)\right] \Sigma_{21}^{z}(q)+J_{\mathbf{q}}^{z} \Sigma_{11}^{z}(q) \Sigma_{22}^{z}(q)}{\left[1-J_{\mathbf{q}}^{z} \Sigma_{12}^{z}(q)\right]\left[1-J_{\mathbf{q}}^{z} \Sigma_{21}^{z}(q)\right]-\left(J_{q}^{z}\right)^{2} \Sigma_{11}^{z}(q) \Sigma_{22}^{z}(q)} .
$$

The expressions for the functions $G_{22}^{z z}(q)$ and $G_{12}^{z z}(q)$ can be obtained from Eqs. (5) and (6), respectively, by substitutions $1 \rightarrow 2 \rightarrow 1$.

Summing up the results, we obtain a general expression $G_{\text {tot }}^{z z}(q)=N(q) / D(q)$. Here, we specify the numerator as

$$
\begin{aligned}
& N(q)=\left\{\Sigma_{11}^{z}(q)+\Sigma_{22}^{z}(q)-\left[\Sigma_{12}^{z}(q)+\Sigma_{21}^{z}(q)-\right.\right. \\
& \left.\left.-2 J_{q}^{z} \Sigma_{12}^{z}(q) \Sigma_{21}^{z}(q)+2 J_{\mathbf{q}}^{z} \Sigma_{11}^{z}(q) \Sigma_{22}^{z}(q)\right]\right\}
\end{aligned}
$$

and the denominator as

$$
D(q)=\left[1-J_{q}^{z} \Sigma_{12}^{z}(q)\right]\left[1-J_{q}^{z} \Sigma_{21}^{z}(q)\right]-
$$$$
-\left(J_{q}^{z}\right)^{2} \Sigma_{11}^{z}(q) \Sigma_{22}^{z}(q) \text {. }
$$

The graphical representations of the irreducible parts $\Sigma_{i j}^{z}(q)$ allow us to summing up the infinite series of relative diagrams. This gives the possibility to obtain an expression for the longitudinal spin susceptibility $\chi^{z z}(\mathbf{q}, \omega)$ applicable in all regions of the frequency $\omega$ and wave vector $\mathbf{q}$ spaces beyond the hydrodynamical and critical regimes. Longitudinal GFs' irreducible parts $\Sigma_{i j}^{z}(q)$ consist of a series of different 

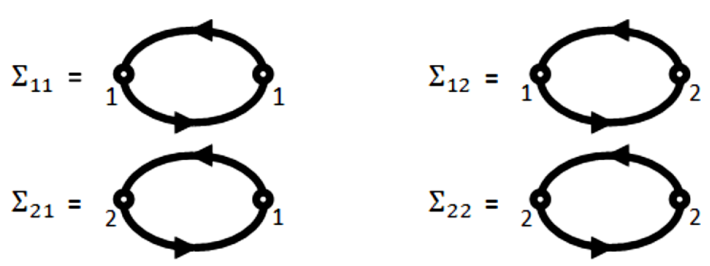

Fig. 2. One-loop order of the graphical presentation of longitudinal spin GFs' irreducible parts

types of loops. To sum up, the series a random-phase approximation (RPA) is typically used $[21,22]$. It can be shown that, in the zeroth order of a large interaction radius (or of the zeroth order of the parameter $1 / n_{0}$, where $n_{0}$ is the number of nearest neighbors), one obtains $\Sigma_{11}^{z}(q)=\delta_{n, 0} b^{\prime}, \Sigma_{22}^{z}(q)=\delta_{n, 0} b^{\prime}$, $\Sigma_{12}^{z}(q)=\Sigma_{21}^{z}(q)=0$ (here, $b^{\prime}$ stands for the first derivative of the Brillouin function, and $\delta_{n, 0}=\delta_{\omega_{n}, 0}$ is the Kronecker symbol for the corresponding frequency difference).

Note that, within the zero-order approximation, we deal with static fluctuations of the longitudinal spin components, which are characterized by the derivatives of the Brillouin functions $\left(\sim \delta_{n, 0} b^{\prime}\right)$ and are responsible for the distinction between the isolated and isothermal susceptibilities of the system. We are interested in isolated system's susceptibility, and these diagrams are not shown in Fig. 1. Note in this regard that the fluctuation relaxation of SWs in a uniaxial AFM near the Néel temperature was investigated theoretically in work [23]. The obtained results showed that the relaxation rate of magnons vanishes due to fluctuation scattering, i.e., $\sim \delta_{n, 0} b^{\prime}$, at $\mathbf{q} \rightarrow 0$. Let us emphasize in this relation that the traditional representation of spin operators by Bose operators (e.g., the Holstein-Primakov or Dyson-Maleev representations, see, e.g. [24]) account only for the dynamic fluctuations in the magnetization, i.e., a reduction in the magnetization of the sublattice owing to the thermal excitation of SWs. Static fluctuations of the longitudinal components of spins are entirely neglected in terms of these representations.

To restore the dynamical characteristics, we need to calculate the irreducible parts within non-zeroth approximations. The graphs for the "uncuttable" parts $\Sigma_{i j}^{z}(q)$ in the one-loop order approximation are shown in Fig. 2. [Recall that we are interested in the Kubo (or isolated) susceptibility of the system obtained from the $G_{\text {tot }}^{z z}\left(q, i \omega_{n}\right)$ by analytic continuation from the Matsubara frequencies onto the real axis $i \omega_{n} \rightarrow$ $\rightarrow \omega+i \delta(\delta \rightarrow 0)$. By this reason, the diagrams which do not depend on the thermodynamic time (i.e., $\sim \delta_{n, 0}$ ) in Fig. 2 are not shown.] In the figure, the thick lines represent the "dressed" transverse GFs, $G_{i j}(q)$, and the hollow points indicate vertices corresponding to the operators $S_{i}^{z}(i=1,2)$. The related analytic expression for $\Sigma_{i j}^{z}(q)$ is:

$\Sigma_{i j}^{(1)}=N^{-1} \beta^{-1} \sum_{p} G_{i j}(p) G_{j i}(p-q)$.

The dressed lines of transverse GFs of SWs are a result of the graphical series summation for the transverse GFs (see Sec. 4). Following the rules of diagram techniques, one can obtain the analytic expressions for GF irreducible parts. For the diagrams in Fig. 2, we write the result in the form: $\Sigma_{11}^{z(1)}(q)=$ $=\Pi(q)+\left(\sim \delta_{n, 0}\right), \Sigma_{12}^{z(1)}(q)=\Sigma_{21}^{z(1)}(q)=\Phi(q)+$ $+\left(\sim \delta_{n, 0}\right)$, and $\Sigma_{22}^{z(1)}(q)=B(q)+\left(\sim \delta_{n, 0}\right)$. Here, $\left(\sim \delta_{n, 0}\right)$ stands for the analytic expressions of the diagrams which do not contribute into isolated system's susceptibilities (these diagrams are not shown in Fig. 2 explicitly). Then the contribution of oneloop graphs to the longitudinal GFs are

$$
\begin{aligned}
& \Pi(q)=N^{-1} \beta^{-1} \sum_{p} G_{11}(p) G_{11}(p-q), \\
& \Phi(q)=N^{-1} \beta^{-1} \sum_{p} G_{12}(p) G_{21}(p-q), \\
& B(q)=N^{-1} \beta^{-1} \sum_{p} G_{22}(p) G_{22}(p-q) .
\end{aligned}
$$

The series of one-loop order diagrams for SW propagators are summed up in Ref. [20].

All possible two-loop diagrams which are related to the Kubo (isolated) susceptibility are depicted in Fig. 3. The hatched squares represent graphically the effective four-point vertices $\Gamma_{i i, i j}\left(k_{1}, k_{2} \mid k_{1}-q, k_{2}+q\right)$. The equations for the effective four-point vertices $\Gamma_{i i, i j}\left(k_{1}, k_{2} \mid k_{1}-q, k_{2}+q\right)$ are presented graphically in Fig. 4 . The analytic expression for the vortex $\Gamma_{11,12}\left(k_{1}, k_{2} \mid k_{1-q}, k_{2+q}\right)$ that is shown in Fig. 4, a looks like:

$$
\begin{aligned}
& \Gamma_{11,12}\left(k_{1}, k_{2} \mid k_{1}-q, k_{2}+q\right)=J_{\mathbf{k}_{2}+\mathbf{q}}+ \\
& +\frac{1}{\beta N} \sum_{k_{3}} J_{\mathbf{k}_{3}+\mathbf{q}} G_{11}\left(k_{3}\right) G_{21}\left(k_{3}+q\right) \times \\
& \times \Gamma_{11,12}\left(k_{3}+q, k_{2} \mid k_{3}, k_{2}+q\right) .
\end{aligned}
$$

ISSN 2071-0194. Ukr. J. Phys. 2020. Vol. 65, No. 10 
If we multiply both sides by $J_{\mathbf{k}_{1}} G_{11}\left(k_{1}-q\right) G_{21}\left(k_{1}\right)$ and then sum up over $k_{1}$, this results in a linear relation, and we get explicitly:

$\Gamma_{11,12}\left(k_{1}, k_{2} \mid k_{1}-q, k_{2}+q\right)=\frac{J_{\mathbf{k}_{2}+\mathbf{q}}}{1-Q(q)}$,

where $Q(q)=\frac{1}{\beta N} \sum J_{\mathbf{p}} G_{11}(p-q) G_{21}(p)$. The analytic expression for the vortex $\Gamma_{22,21}\left(k_{1}, k_{2} \mid k_{1}-q, k_{2}+q\right)$ that is shown in Fig. 4, $b$ looks like:

$\Gamma_{22,21}\left(k_{1}, k_{2} \mid k_{1}-q, k_{2}+q\right)=J_{\mathbf{k}_{2}+\mathbf{q}}+$

$+\frac{1}{\beta N} \sum_{k_{3}} J_{\mathbf{k}_{3}+\mathbf{q}} G_{22}\left(k_{3}\right) G_{12}\left(k_{3}+q\right) \times$

$\times \Gamma_{22,21}\left(k_{3}+q, k_{2} \mid k_{3}, k_{2}+q\right)$.

Multiplying both sides by $J_{\mathbf{k}_{1}} G_{22}\left(k_{1}-q\right) G_{12}\left(k_{1}\right)$ and then summing up over $k_{1}$, we get

$\Gamma_{22,21}\left(k_{1}, k_{2} \mid k_{1}-q, k_{2}+q\right)=\frac{J_{\mathbf{k}_{2}+\mathbf{q}}}{1-\Lambda(q)}$,

where $\Lambda(q)=\frac{1}{\beta N} \sum J_{\mathbf{p}} G_{22}(p-q) G_{12}(p)$. The expressions of another two vertices have a similar form:

$\Gamma_{22,12}\left(k_{1}, k_{2} \mid k_{1}-q, k_{2}+q\right)=\frac{J_{\mathbf{k}_{1}-q}}{1-\Lambda(q)}$,

$\Gamma_{11,21}\left(k_{1}, k_{2} \mid k_{1}-q, k_{2}+q\right)=\frac{J_{\mathbf{k}_{1}-q}}{1-Q(q)}$.

Using the obtained expressions for the vertices and summing up all contributions, we get the following analytic expressions for the two-loop order diagrams for irreducible parts that are shown in Fig. 3:

$\Sigma_{22}^{z(2)}=2\left(\frac{\Lambda B}{1-\Lambda}+\frac{\Lambda^{\prime} \Phi}{1-Q}\right)$

$\Sigma_{11}^{z(2)}=2\left(\frac{Q^{\prime} \Phi}{1-\Lambda}+\frac{\Pi Q}{1-Q}\right)$,

$\Sigma_{12}^{z(2)}=\Sigma_{21}^{z(2)}=\frac{Q^{\prime} B+\Phi \Lambda}{1-\Lambda}+\frac{Q \Phi+\Lambda^{\prime} \Pi}{1-Q}$,

where $\Lambda^{\prime}(q)=\frac{1}{\beta N} \Sigma J_{\mathbf{p}} G_{22}(p) G_{12}(p-q)$ and $Q^{\prime}(q)=$ $=\frac{1}{\beta N} \Sigma J_{\mathbf{p}} G_{11}(p) G_{21}(p-q)$. The total contributions of the irreducible parts are the sum of one-loop and two-loop orders: $\Sigma_{22}^{z}=\Sigma_{22}^{z(1)}+\Sigma_{22}^{z(2)}$. Thus, to find the isolated susceptibility $\chi^{z z}(q)$, it is necessary to calculate $\Pi(q), \Phi(q), B(q), \Lambda(q), \Lambda^{\prime}(q), Q(q), Q^{\prime}(q)$ which of them consists of transverse GFs.

ISSN 2071-0194. Ukr. J. Phys. 2020. Vol. 65, No. 10

\section{Transverse Green Functions}

Dealing with transverse SW excitations, we are interested in the summation of graphical series for the GFs in the Hartree-Fock approximation. In this approximation, the transverse $G_{i j}(q)$ can be presented explicitly in the following form (for details, see [20]):

$\left\{\begin{array}{l}G_{11}(q)=b K_{11}+b K_{11} J_{12} G_{21}(q), \\ G_{21}(q)=-b K_{22} J_{12} G_{11}(q) .\end{array}\right.$
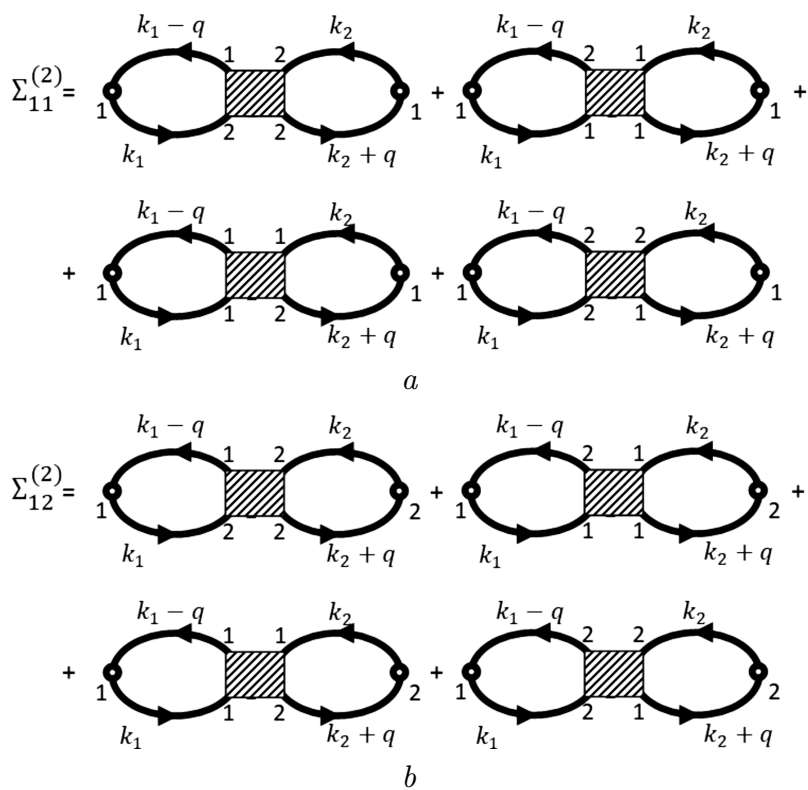

Fig. 3. Two-loop order of the graphical presentation of longi-tudinal spin GFs' irreducible parts. The hatched squares represent graphically the effective four-point vertices $\Gamma_{i i, i j}\left(k_{1}, k_{2} \mid k_{1}-q, k_{2}+q\right)$
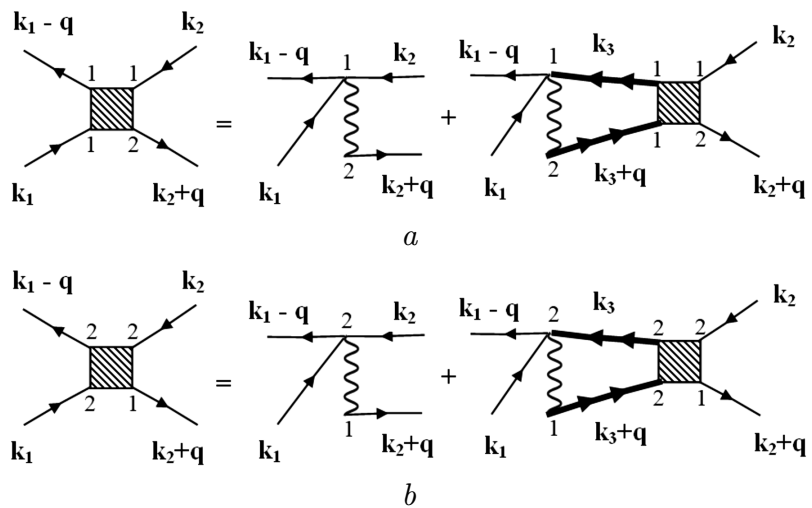

Fig. 4. Graphical presentation of the effective fourpoint vertices: $\quad \Gamma_{11,12}\left(k_{1}, k_{2} \mid k_{1}-q, k_{2}+q\right) \quad(a) \quad$ and $\Gamma_{22,21}\left(k_{1}, k_{2} \mid k_{1}-q, k_{2}+q\right)(b)$ 
The system of equations for the transverse $G_{22}(q)$ and $G_{12}(q)$ GFs possesses a similar structure. Here, $K_{11}=K_{11}\left(i \omega_{n}\right)=1 /\left(i \omega_{n}+y_{0}\right), K_{22}=K_{22}\left(i \omega_{n}\right)=$ $=1 /\left(i \omega_{n}-y_{0}\right)$, and $y_{0}=b J_{0}^{z}$. Explicit expressions for the transversal GFs $G_{i j}(q)$ are given by:

$$
\begin{aligned}
& G_{11}(q)=\frac{b K_{22}^{-1}}{\left(i \omega_{n}+\varepsilon_{\mathbf{q}}\right)\left(i \omega_{n}-\varepsilon_{\mathbf{q}}\right)}, \\
& G_{12}(q)=G_{21}(q)=-\frac{b^{2} J_{\mathbf{q}}}{\left(i \omega_{n}+\varepsilon_{\mathbf{q}}\right)\left(i \omega_{n}-\varepsilon_{\mathbf{q}}\right)}, \\
& G_{22}(q)=-\frac{b K_{11}^{-1}}{\left(i \omega_{n}+\varepsilon_{\mathbf{q}}\right)\left(i \omega_{n}-\varepsilon_{\mathbf{q}}\right)} .
\end{aligned}
$$

Since SW's spectrum of the AFM is degenerate, we used, here and below, the designation $\varepsilon(\mathbf{q})=\varepsilon_{\mathbf{q}}$.

The main physics can be captured in a long-wave limit $(a q) \ll 1$ (here, $a$ stands for the lattice spacing). Within this approximation, the energy of transverse SW excitations reads

$\varepsilon_{\mathbf{q}}=\sqrt{\Delta^{2}+(D q)^{2}}$,

where $D=b \sqrt{2 J_{0} J}, \Delta=b \sqrt{\left(J_{0}^{z}\right)^{2}-\left(J_{0}\right)^{2}}$. Here, we used the quadratic expansion, when evaluating the quantity $J_{0}-J_{\mathbf{q}} \sim J(a q)^{2}=J_{0}(a q)^{2} / n_{0}$.

\section{Longitudinal Spin Dynamics}

The excitation spectrum of the two-sublattice AFM is determined by the poles of the analytically continued temperature GF $G_{\text {tot }}^{z z}\left(\mathbf{q}, i \omega_{n}\right) i \omega_{n} \rightarrow \omega+i \delta(\delta \rightarrow 0)$ $[21,22]$. The real part of the pole is the energy of a quasiparticle excitation, while the imaginary part characterizes the broadening of this energy level, i.e., a quasiparticle damping. Thus, we should investigate whether the equation $D\left(\mathbf{q}, i \omega_{n} \rightarrow \omega+i \delta\right)=0$, $(\delta \rightarrow 0)$, for the denominator of $G_{\text {tot }}^{z z}\left(\mathbf{q}, i \omega_{n}\right)$ has solutions that would determine the longitudinal wave excitations. To this end, let us examine expression (8) more closely. Within the two-loop approximation, the denominator in Eq. (8) can be rewritten in the form

$$
\begin{aligned}
& D(q)=1-2 J_{\mathbf{q}}^{z}\left(\Sigma_{12}^{(1)}+\Sigma_{12}^{(2)}-\Sigma_{12}^{(1)} \Sigma_{12}^{(2)}\right)+\left(J_{\mathbf{q}}^{z} \Sigma_{12}^{(1)}\right)^{2}- \\
& -\left(J_{\mathbf{q}}^{z}\right)^{2}\left[\Sigma_{11}^{(1)} \Sigma_{22}^{(1)}+\Sigma_{11}^{(1)} \Sigma_{22}^{(2)}+\Sigma_{11}^{(2)} \Sigma_{22}^{(1)}\right] .
\end{aligned}
$$

The quantities $\Sigma_{i j}^{(1)}$ and $\Sigma_{i j}^{(2)}$ are presented by Eqs. (10)-(12) and Eqs. (17)-(19), respectively. In
Eq. (24), we skip the term $\Sigma_{11}^{(2)} \Sigma_{22}^{(2)}$, as it is of a higherorder approximation. After summing up over the discrete Matsubara frequency for the function $\Phi(q)=$ $=\Phi\left(\mathbf{q}, i \omega_{n}\right)$, Eg. (11), we obtain

$$
\begin{aligned}
& \Phi(q)=\frac{b^{4}}{4 N} \sum_{p} \frac{J_{\mathbf{p}}}{\varepsilon_{\mathbf{p}}} \frac{J_{\mathbf{p}-\mathbf{q}}}{\varepsilon_{\mathbf{p}-\mathbf{q}}}\left\{\frac{n\left(\varepsilon_{\mathbf{p}}\right)-n\left(\varepsilon_{\mathbf{p}-\mathbf{q}}\right)}{i \omega_{q}-\varepsilon_{\mathbf{p}}+\varepsilon_{\mathbf{p}-\mathbf{q}}}-\right. \\
& -\frac{n\left(\varepsilon_{\mathbf{p}}\right)-n\left(\varepsilon_{\mathbf{p}-\mathbf{q}}\right)}{i \omega_{q}+\varepsilon_{\mathbf{p}}-\varepsilon_{\mathbf{p}-\mathbf{q}}}+\frac{1+n\left(\varepsilon_{\mathbf{p}-\mathbf{q}}\right)+n\left(\varepsilon_{\mathbf{p}}\right)}{i \omega_{q}+\varepsilon_{\mathbf{p}}+\varepsilon_{\mathbf{p}-\mathbf{q}}}- \\
& \left.-\frac{1+n\left(\varepsilon_{\mathbf{p}}\right)+n\left(\varepsilon_{\mathbf{p}-\mathbf{q}}\right)}{i \omega_{q}-\varepsilon_{\mathbf{p}}-\varepsilon_{\mathbf{p}-\mathbf{q}}}\right\} .
\end{aligned}
$$

Here, $n\left(\varepsilon_{\mathbf{p}}\right)$ is the Bose distribution function for SW excitations. The expressions for the $\Pi(q), B(q)$ and components of $\Sigma_{i j}^{(2)}-\Lambda(q), Q(q), \Lambda^{\prime}(q)$ and $Q^{\prime}(q)-$ have a similar structure. As follows from (25), the dynamics of longitudinal spin components is due to a few virtual processes of creation and annihilation of transverse SW modes. Namely, the first channel the first and second items in the rhs of Eq. (25) - represents the processes of absorption of one transverse magnon and excitation of another one with energies $\varepsilon_{\mathbf{p}}$ and $\varepsilon_{\mathbf{p}-\mathbf{q}}$, respectively. This channel is controlled by the thermal occupation factors $n\left(\varepsilon_{\mathbf{p}}\right)$, which makes the SWs with $\mathbf{p} \sim 0$ to be dominant.

There is also the second channel - the remaining terms in the Eq. (25). Namely, there is the twoSW creation/annihilation process at the frequency $\omega(\mathbf{q})=\varepsilon_{\mathbf{p}}+\varepsilon_{\mathbf{p}-\mathbf{q}}$. This channel remains even in the absence of thermal excitations, i.e., when $n\left(\varepsilon_{\mathbf{p}}\right) \sim 0$ and/or $n\left(\varepsilon_{\mathbf{p}-\mathbf{q}}\right) \sim 0$. Thus, the structure of the denominator $D(\mathbf{q}, \omega)$ and, in particular, the equation $\operatorname{Re} D(\mathbf{q}, \omega)=0$ that determines the dispersion law indicate a strong renormalization of the frequency of longitudinal spin excitations due to the processes of creation/annihilation of a few virtual transverse SWs.

Let us now examine (24) more closely. Due to a rather complex dependence of the function on the frequency, we consider the real and imaginary parts of $D(\mathbf{q}, \omega)$ only in some limiting cases. Namely, we investigate the function near the singularities of $\Pi(q)$, $\Phi(q), B(q), \Lambda(q), Q(q), \Lambda^{\prime}(q)$, and $Q^{\prime}(q)$, when the equations $\pm \omega_{\|}^{(-)}(\mathbf{q})=\varepsilon_{\mathbf{p}}-\varepsilon_{\mathbf{p}-\mathbf{q}}$ or $\pm \omega_{\|}^{(+)}(\mathbf{q})=\varepsilon_{\mathbf{p}}+$ $+\varepsilon_{\mathbf{p}-\mathbf{q}}$ are fulfilled, i.e., when the related virtual twoSW processes are most effective. From $\operatorname{Re} D(q)=0$, we can obtain the resonance frequencies, and, from $\operatorname{Im} D(q)$, we can find the damping term. We suggest 
that $T \rightarrow T_{N}$ ( $T_{N}$ is the Néel temperature), i.e., the sublattice magnetization $b(T) \ll S$. We also consider the case of small anisotropy, i.e., $\Delta \ll D$.

\subsection{Exchange Longitudinal Excitation}

Let us start with considering the exchange channel, namely, a two-SW creation/annihilation process. At $\omega_{\|}^{(+)}(\mathbf{q})=\varepsilon_{\mathbf{p}}+\varepsilon_{\mathbf{p}-\mathbf{q}}$, the equation for the longitudinal SW excitation gives (for more details, see Appendix A):

$\omega_{\|}^{(+)}(\mathbf{q}) \sim \sqrt{4 \Delta^{2}+\frac{2}{9} q^{2} D^{2}+\frac{4 q \Delta D J_{0}}{3 \sqrt{2} J_{0}^{z}}}$.

Thus, the frequency of a longitudinal spin excitation of this type lies energetically above the transverse SW frequency $\varepsilon_{\mathbf{q}}(23)$ and, at $\mathbf{q} \rightarrow 0$, demonstrates a linear dependence on the wave vector $\omega_{\|}^{(+)}(\mathbf{q}) \sim 2 \Delta+$ $+\frac{q D J_{0}}{6 \sqrt{2} J_{0}^{z} \Delta}$. As was already mentioned, this channel remains in force even in the absence of thermal excitations. One can show that the overall scattering weight of the simultaneous creation/annihilation of two magnons is proportional to the zero-point longitudinal quantum fluctuations in the ground state [24].

The damping coefficient of this channel depends on the wave vector $q$ and the temperature:

$\gamma(\mathbf{q}) \sim T b q^{2} C_{0}$

where $C_{0}=\frac{1}{3(2 \pi)^{2}} \sqrt{\frac{J_{0}}{J}}\left(\frac{2\left(J_{0}^{z}\right)^{2}}{\left(J_{0}^{z}\right)^{2}-\left(J_{0}\right)^{2}}-1\right)$.

\subsection{Acoustic Longitudinal Excitation}

To find the frequency of the acoustic-type channel creation of one magnon and annihilation of another one - we also need to solve $\operatorname{Re} D(q)=0$ at $\omega_{\|}^{(-)}(\mathbf{q})=$ $=\varepsilon_{\mathbf{p}}-\varepsilon_{\mathbf{p}-\mathbf{q}}$. In this case we get:

$\omega_{\|}^{(-)}(\mathbf{q}) \sim b q\left(J_{0}^{z}\right)^{2} C_{1} \sqrt{\frac{T}{\Delta}}$,

where

$C_{1}=\frac{1}{4} \sqrt{\frac{3 J_{0}^{z} b}{\pi\left(6\left(J_{0}^{z}\right)^{2}-J_{0} J\right) \sqrt{2 J_{0} J}}}$.

This channel is controlled by the thermal occupation factor of magnons, which makes the SWs with the wave vector $\mathbf{q} \rightarrow 0$ to be dominant. The frequency of these longitudinal spin excitations is linear in the wave vector and lies energetically below the transverse SW frequency $\varepsilon_{\mathbf{q}}$, Eg. (23), at the same temperature and wave vector. The damping of this channel also has a linear dependence on the wave vector $q$ :

$\gamma(\mathbf{q})=q b J_{0}^{z}\left(\frac{T}{2 J_{0} J}\right)^{3 / 2} C_{2}$

where

$C_{2}=\frac{\sqrt{\Delta}}{2(2 \pi)^{3} C_{1}}\left[\ln \left(\frac{2 D}{\Delta}\right)-1\right]\left[\frac{\left(b J_{0}^{z}\right)^{2}}{D}\left(\frac{3 \pi}{8 \Delta}-\frac{5}{4 D}\right)+1\right]$.

Thus, a feature of the longitudinal excitations in AFM is their similarity with the longitudinal dynamics in a ferrimagnet. Comparing expressions (23) with (26) and (28), one can see that the frequency of the exchange-type longitudinal excitation is above the energy of the transverse one, while the energy of the acoustic longitudinal excitation lies below the energy of a transverse SW. As is seen from Egs. (27) and (30), the relaxation frequency due to the scattering of SWs vanishes at $\mathbf{q} \rightarrow 0$.

\section{Discussions}

An experimental study of the longitudinal magnetization dynamics of the AFM was carried out using the polarized neutron scattering in $[18,25]$. The analysis of the experimental data on the scattering in $\mathrm{RbMnF}_{3}$ showed the presence of an additional contribution related to longitudinal fluctuations [25]. The authors of work [25] showed that the longitudinal contribution intensity decreases with increasing the wave vector and with decreasing the temperature below $T_{N}$.

The separation between the longitudinal and transverse components of the magnons spectrum was also found in the polarization analysis of neutron scattering experiments in anisotropic AFM $\mathrm{MnF}_{2}$ [18]. The main difference is in the characters of excitations: in the longitudinal case, these are two-magnon processes, while the transverse excitation is related to one-magnon processes. Moreover, it was shown that the longitudinal mode is divided into two regions: two-magnon excitation (absorption) and magnon scattering (creation of one magnon and annihilation of another one), which separated by the energy gap.

The authors of work [19] investigated anisotropic AFM $\mathrm{MnF}_{2}$. They identified a set of two-SW annihilation and creation peaks. For the isotropic AFM, 
they also found the presence of both two-SW creation/annihilation peaks, as well as the creation of one SW and the annihilation of another one. When $T$ approaches $T_{N}$, the two-SW peaks disappear into the tails of the one-SW peak.

Like the works $[18,19]$, our theoretical analysis has shown that, in an AFM, the longitudinal dynamics is generated by the creation and annihilation of two SWs. The peculiarity of the longitudinal spin dynamics lies in the specific quantum properties of spin operators [24]. Namely, the commutator of the one-SW creation (annihilation) operators $S^{+}$ and $S^{-}$is the operator $S^{z}$ that is not a $c$-number. As a result, the longitudinal vibrations of the magnetization caused by an external magnetic field, $h_{z} S^{z}$, may be realized only as the virtual processes of coherent creation and annihilation of two transverse SWs: $h_{z} S^{z} \sim h_{z}\left(S^{+} S^{-}\right)$.

From the mathematical point of view, the study of the longitudinal dynamics is reduced to summing up all the loop diagrams describing two-magnon processes. The series of the related loop diagrams turns out to be rather complicated. The first-order approximation contains four different types of loops $\Sigma_{i j}^{z z}$. The results of studies taking this order into account can be found in Ref. [20].

The second-order approximation is more complicated and involves finding not only the first-order loops, but the interaction vertices $\Gamma_{i i, i j}\left(k_{1}, k_{1}-\right.$ $\left.-q \mid k_{2}, k_{2}+q\right)$ as well. To sum up these series, we have used a method called the generalized RPA elaborated earlier $[9,23,24]$. Using the two-loop order approximation, we obtained the longitudinal retarded GFs. The analytic continuation of GFs onto the real frequency axis allowed us to determine the longitudinal susceptibility $\chi^{z z}(\mathbf{q}, \omega)$ of the AFM. The susceptibility was examined as a function of the frequency $\omega$ and the wave vector $\mathbf{q}$.

Thus, we have shown that the longitudinal susceptibility $\chi^{z z}(\mathbf{q}, \omega)$ of an AFM contains two types of longitudinal excitations. These excitations have different dependences on the temperature and the wave vector. It is validated that the frequency of one type of longitudinal excitations lies above the transverse SW frequency, while the second type of excitations lies below the transverse SW frequency. The process of two-magnon excitation (absorption) remains even in the absence of thermal excitations. In our opinion, it provides most likely the main contribution to the thermally induced longitudinal magnetization dynamics.

V.K. gratefully acknowledges the numerous fruitful discussions of various questions of magnetism with S.M. Ryabchenko.

\section{APPENDIX A}

In order to find the frequency and the damping of the creation or annihilation of two magnons, it is necessary to find the real and imaginary parts of the denominator $D(q)$. As the frequencies of two processes can be separated, we operate with terms that correspond only to a given channel. For $\omega_{\|}^{(+)}(\mathbf{q})=\varepsilon_{\mathbf{p}}+\varepsilon_{\mathbf{p}-\mathbf{q}}$, we get the following expressions:

$\Phi(q) \approx \frac{b^{4}}{4 N} \sum_{p}\left(\frac{J_{\mathbf{p}}}{\varepsilon_{\mathbf{p}}}\right)^{2}\left(1+n_{p}+n_{p-q}\right) \times$

$\times\left\{\frac{1}{i \omega_{q}+\varepsilon_{\mathbf{p}}+\varepsilon_{\mathbf{p}-\mathbf{q}}}-\frac{1}{i \omega_{q}-\varepsilon_{\mathbf{p}}-\varepsilon_{\mathbf{p}-\mathbf{q}}}\right\}$,

$\Lambda^{\prime}(q)=Q(q) \approx-\frac{b^{3}}{4 N} \sum_{p}\left(\frac{J_{\mathbf{p}}}{\varepsilon_{\mathbf{p}}}\right)^{2}\left(1+n_{p}+n_{p-q}\right) \times$

$\times\left\{\frac{\varepsilon_{\mathbf{p}}-b J_{0}^{z}}{i \omega_{q}+\varepsilon_{\mathbf{p}}+\varepsilon_{\mathbf{p}-\mathbf{q}}}+\frac{\varepsilon_{\mathbf{p}}+b J_{0}^{z}}{i \omega_{q}-\varepsilon_{\mathbf{p}}-\varepsilon_{\mathbf{p}-\mathbf{q}}}\right\}$,

$Q^{\prime}(q)=\Lambda(q) \approx \frac{b^{3}}{4 N} \sum_{p}\left(\frac{J_{\mathbf{p}}}{\varepsilon_{\mathbf{p}}}\right)^{2}\left(1+n_{p}+n_{p-q}\right) \times$

$\times\left\{\frac{\varepsilon_{\mathbf{p}-\mathbf{q}}+b J_{0}^{z}}{i \omega_{q}+\varepsilon_{\mathbf{p}}+\varepsilon_{\mathbf{p}-\mathbf{q}}}+\frac{\varepsilon_{\mathbf{p}-\mathbf{q}}-b J_{0}^{z}}{i \omega_{q}-\varepsilon_{\mathbf{p}}-\varepsilon_{\mathbf{p}-\mathbf{q}}}\right\}$.

In this case $\Phi(q)=\Pi(q)=B(q)$, and considering the fact that $\Lambda+Q=2 J_{0}^{z} \Phi$ the equation for $D(q)$ transforms into: $D(q)=1-2 \Phi\left(J_{0}^{z}+J_{\mathbf{q}}^{z}\right)$. Thus, to find the energy of longitudinal excitations $\omega_{\|}^{(+)}(\mathbf{q})$, we need to solve the equation

$1-2\left(J_{0}^{z}+J_{\mathbf{q}}^{z}\right) \operatorname{Re} \Phi(q)=0$.

After that, we find the damping coefficient of this excitation as $\gamma(\mathbf{q})=-2\left(J_{0}^{z}+J_{\mathbf{q}}^{z}\right) \operatorname{Im} \Phi\left(\omega=\omega_{\|}^{(+)}\right)$.

\section{APPENDIX B}

In order to describe the process of creation-annihilation of SWs, $\omega_{\|}^{(-)}(\mathbf{q})=\varepsilon_{\mathbf{p}}-\varepsilon_{\mathbf{p}-\mathbf{q}}$, we need to find the real and imaginary parts of $D(q)$. Thus, we need to calculate the items in $\Phi(q), \Pi(q), B(q), \Lambda(q), \Lambda(q)^{\prime}, Q(q)$, and $Q(q)^{\prime}$ that match this channel. In this case, $\Phi(q) \neq \Pi(q) \neq B(q)$ and looks like

$$
\begin{aligned}
& \Phi(q) \approx \frac{b^{4}}{4 N} \sum_{p}\left(\frac{J_{\mathbf{p}}}{\varepsilon_{\mathbf{p}}}\right)^{2}\left(n_{p}-n_{p-q}\right) \times \\
& \times\left\{\frac{1}{i \omega_{q}-\varepsilon_{\mathbf{p}}+\varepsilon_{\mathbf{p}-\mathbf{q}}}-\frac{1}{i \omega_{q}+\varepsilon_{\mathbf{p}}-\varepsilon_{\mathbf{p}-\mathbf{q}}}\right\},
\end{aligned}
$$




$$
\begin{aligned}
& \Pi(q) \approx \frac{b^{2}}{4 N} \sum_{p} \frac{1}{\varepsilon_{\mathbf{p}}^{2}}\left(n_{p}-n_{p-q}\right) \times \\
& \times\left\{\frac{\left(\varepsilon_{\mathbf{p}}-b J_{0}^{z}\right)^{2}}{i \omega_{q}-\varepsilon_{\mathbf{p}}+\varepsilon_{\mathbf{p}-\mathbf{q}}}-\frac{\left(\varepsilon_{\mathbf{p}}+b J_{0}^{z}\right)^{2}}{i \omega_{q}+\varepsilon_{\mathbf{p}}-\varepsilon_{\mathbf{p}-\mathbf{q}}}\right\}, \\
& B(q) \approx \frac{b^{2}}{4 N} \sum_{p} \frac{1}{\varepsilon_{\mathbf{p}}^{2}}\left(n_{p}-n_{p-q}\right) \times \\
& \times\left\{\frac{\left(\varepsilon_{\mathbf{p}}+b J_{0}^{z}\right)^{2}}{i \omega_{q}-\varepsilon_{\mathbf{p}}+\varepsilon_{\mathbf{p}-\mathbf{q}}}-\frac{\left(\varepsilon_{\mathbf{p}}-b J_{0}^{z}\right)^{2}}{i \omega_{q}+\varepsilon_{\mathbf{p}}-\varepsilon_{\mathbf{p}-\mathbf{q}}}\right\} .
\end{aligned}
$$

The expressions for components of $\Sigma_{i j}^{(2)}$ are:

$$
\begin{aligned}
& Q^{\prime}=Q \approx-\frac{b^{3}}{4 N} \sum_{p}\left(\frac{J_{\mathbf{p}}}{\varepsilon_{\mathbf{p}}}\right)^{2}\left(1+n_{p}+n_{p-q}\right) \times \\
& \times\left\{\frac{\varepsilon_{\mathbf{p}}-b J_{0}^{z}}{i \omega_{q}-\varepsilon_{\mathbf{p}}+\varepsilon_{\mathbf{p}-\mathbf{q}}}+\frac{\varepsilon_{\mathbf{p}}+b J_{0}^{z}}{i \omega_{q}+\varepsilon_{\mathbf{p}}-\varepsilon_{\mathbf{p}-\mathbf{q}}}\right\}, \\
& \Lambda^{\prime}=\Lambda \approx \frac{b^{3}}{4 N} \sum_{p}\left(\frac{J_{\mathbf{p}}}{\varepsilon_{\mathbf{p}}}\right)^{2}\left(1+n_{p}+n_{p-q}\right) \times \\
& \times\left\{\frac{\varepsilon_{\mathbf{p}-\mathbf{q}}+b J_{0}^{z}}{i \omega_{q}-\varepsilon_{\mathbf{p}}+\varepsilon_{\mathbf{p}-\mathbf{q}}}+\frac{\varepsilon_{\mathbf{p}-\mathbf{q}}-b J_{0}^{z}}{i \omega_{q}+\varepsilon_{\mathbf{p}}-\varepsilon_{\mathbf{p}-\mathbf{q}}}\right\} .
\end{aligned}
$$

As for the previous channel, we have $\Lambda+Q=2 J_{0}^{z} \Phi$. Substituting the expressions for $\Sigma_{i j}^{z}$ in Eq. (24), we get the equation for finding $\omega_{\|}^{(-)}(\mathbf{q})$ :

$1-2 J_{0}^{z} \Phi-2 J_{\mathbf{q}}^{z}(\Phi+Q B+\Pi \Lambda)-$

$-\left(J_{\mathbf{q}}^{z}\right)^{2} \Pi B\left[1+2 J_{0}^{z} \Phi\right]=0$.

After some transformations and calculations of the real and imaginary parts for Eqs. (B1)-(B5), we find that $\operatorname{Re} \Pi=\operatorname{Re} B$, $\operatorname{Re} \Lambda=\operatorname{Re} Q, \operatorname{Im} \Pi=-\operatorname{Im} B, \operatorname{Im} Q==-\operatorname{Im} \Lambda$. So, the real part of the denominator transforms into:

$$
\begin{aligned}
& 1-2 J_{0}^{z} \operatorname{Re} \Phi-2 J_{\mathbf{q}}^{z}(\operatorname{Re} \Phi+2 \operatorname{Im} Q \operatorname{Im} \Pi)- \\
& -\left(J_{\mathbf{q}}^{z}\right)^{2}(\operatorname{Im} \Pi)^{2}\left[1+2 J_{0}^{z} \operatorname{Re} \Phi\right]=0 .
\end{aligned}
$$

Here, we skipped some terms that were of a higher-order approximation. After the substitution of the components $\operatorname{Re} \Phi$, $\operatorname{Im} \Pi, \operatorname{Im} Q$, we get the resulting expression presented in Eq. (28).

After similar cumbersome calculations, we get the damping term for acoustic longitudinal excitations:

$\gamma(\mathbf{q})=2\left(J_{\mathbf{q}}^{z}\right)^{2} \operatorname{Im} B(q) \operatorname{Re} B(q)$.

The final expression for the damping is presented in Eq. (30).

1. E. Beaurepaire, J.C. Merle, A. Daunois, J.Y. Bigot. Ultrafast spin dynamics in ferromagnetic nickel. Phys. Rev. Lett. 76, 4250 (1996).

2. Yu.A. Izyumov, N.I. Chaschin, V.Yu. Yushankhai. Longitudinal spin dynamics in the Heisenberg ferromagnet: Diagrammatic approach. Phys. Rev. B 65, 214425 (2002).

3. M. Fiebig, N.P. Duong, T. Satoh, B.B. Van Aken, K. Miyano, Y. Tomioka, Y. Tokura. Ultrafast magnetization dynamics of antiferromagnetic compounds. J. Phys. D: Appl. Phys. 41, 164005 (2008).

ISSN 2071-0194. Ukr. J. Phys. 2020. Vol. 65, No. 10
4. J.Y. Bigot, M. Vomir, E. Beaurepaire. Coherent ultrafast magnetism induced by femtosecond laser pulses. Nature Phys. 5, 515 (2009).

5. A. Kirilyuk, A.V. Kimel, T. Rasing. Ultrafast optical manipulation of magnetic order. Rev. Mod. Phys. 82, 2731 (2010).

6. B.Y. Mueller, T. Roth, M. Cinchetti, M. Aeschlimann, B. Rethfeld. Driving force of ultrafast magnetization dynamics. New J. Phys. 13, 123010 (2011).

7. I. Radu et al. Transient ferromagnetic-like state mediating ultrafast reversal of antiferromagnetically coupled spins. Nature 472, 205 (2011).

8. V.N. Krivoruchko. Longitudinal spin dynamics in ferrimagnets: Multiple spin wave nature of longitudinal spin excitations. Phys. Rev. B 94, 054434 (2016).

9. V.G. Vaks, A.I. Larkin, S.A. Pikin. Spin waves and correlation functions in a ferromagnetic. Sov Phys. JETP 26, 647 (1968).

10. B.A. Ivanov. Ultrafast spin dynamics and spintronics for ferrimagnets close to the spin compensation point (Review). Low Temp. Phys. 45, 935 (2019).

11. A.V. Kimel et al. Inertia-driven spin switching in antiferromagnets. Nat. Phys. 5, 727 (2009).

12. P. Wadley et al. Electrical switching of an antiferromagnet. Science 351, 587 (2016).

13. A.V. Kimel, A. Kirilyuk, A. Tsvetkov, R.V. Pisarev, T. Rasing. Laser-induced ultrafast spin reorientation in the antiferromagnet $\mathrm{TmFeO}_{3}$. Nature 429, 850 (2004).

14. D. Bossini, A.M. Kalashnikova, R.V. Pisarev, Th. Rasing, A.V. Kimel. Controlling coherent and incoherent spin dynamics by steering the photoinduced energy flow. Phys. Rev. B 89, 060405 (2014).

15. D. Bossini et al. Laser-driven quantum magnonics and terahertz dynamics of the order parameter in antiferromagnets. Phys. Rev. B 100, 024428 (2019).

16. X. Marti et al. Room-temperature antiferromagnetic memory resistor. Nature Mater. 13, 367 (2014).

17. F. Gomory et al. Experimental realization of a magnetic cloak. Science 335, 1466 (2012).

18. W. Schweika, S.V. Maleyev, Th. Brückel, V.P. Plakhty, L.-P. Regnault. Longitudinal spin fluctuations in the antiferromagnet $\mathrm{MnF}_{2}$ studied by polarized neutron scattering. Europhys. Lett 60, 446 (2002).

19. A. Bunker, D.P. Landau. Longitudinal magnetic excitations in classical spin systems. Phys. Rev. Lett. 85, 2601 (1999).

20. O. Boliasova, V. Krivoruchko. Two-magnon longitudinal excitations in the Heisenberg antiferromagnets. 9th International Conference on Nanomaterials: Applications and Properties, (2019).

21. V.G. Baryakhtar, V.N. Krivoruchko, D.A. Yablonskii. Green Functions in the Theory of Magnetism (Naukova Dumka, 1984) (in Russian).

22. V.G. Baryakhtar, B.A. Ivanov, V.N. Krivoruchko, A.G Danilevich. Modern Problems of Magnetization Dynamics: from the Basis to the Ultrafast Relaxation (Himhest, 2013) (in Russian) [ISBN: 978-966-8537-94-3]. 
23. D.A. Garanin, V.S. Lutovinov. High temperature spin wave dynamics of the uniaxial antiferromagnets. Solid State Comm. 44, 1359 (1982).

24. V.N. Krivoruchko. Longitudinal magnetization dynamics in Heisenberg magnets: Spin Green functions approach (Review Article). Low Temp. Phys. 43, 1245 (2017).

25. U.J. Cox, R.A. Cowley, S. Bates, L.D. Cussen. Longitudinal fluctuations in an isotropic antiferromagnet. J. Phys.: Condens. Matter 1, 3031 (1989).

Received 08.07.20

О.О. Болясова, В.М. Криворучко

ПОЗДОВЖНЯ СПІНОВА ДИНАМІКА

У ГАЙЗЕНБЕРГІВСЬКОМУ АНТИФЕРОМАГНЕТИКУ: ДВОМАГНОННІ ЗБУДЖЕННЯ

$\mathrm{P}$ е $з$ ю м е

Розуміння надшвидкої спінової динаміки в магнітно-упорядкованих матеріалах $є$ важливим для усвідомлення основних меж надшвидкої спінової електроніки - магноніки. У роботі на базі мікроскопічної моделі динаміки намагніченості досліджено поздовжню динаміку намагніченості в двопідгратковому антиферомагнетику. Для подолання обмежень, типових для феноменологічних підходів, застосовувалась діаграмна техніка для спінових операторів. Графічні зображення спінових пропагаторів дозволяють врахувати нескінченну серію відмінних діаграм. Ця сума перетворюється в аналітичний вираз для поздовжньої спінової сприйнятливості $\chi^{z z}(\mathbf{q}, \omega)$, який може бути застосовним для всіх частот $\omega$ i хвильового вектора $\mathbf{q}$ в області за межами гідродинамічного та критичного режимів. Було встановлено, що поздовжня динаміка намагніченості складається з двох типів збуджень, які мають різну залежність від температури та хвильового вектора q. Отриманий результат може бути важливим для розуміння фізики нерівноважної магнітної динаміки під впливом надшвидких лазерних імпульсів у антиферомагнітних матеріалах. 